\title{
EL RÉGIMEN DE JORNADA ESCOLAR COMPLETA Y LA PARTICIPACIÓN EN EL JUEGO: UNA POLÍTICA PÚBLICA EN DEUDA CON LOS NIÑNOS Y NIÑAS.
}

Diego Pablo González Lagos ${ }^{1}$, Guisela Escarlet Leal Barriga², Norma Lucía Monsalves Ferrada $^{3}$ y Karina Andrea Salazar Betanzo ${ }^{4}$.

\section{Resumen}

La investigación "El régimen de Jornada Escolar Completa y la Participación en el Juego: Una política pública en deuda con los niños y niñas" muestra el impacto de la Jornada Escolar Completa en la Participación en el luego de escolares de segundo año básico pertenecientes a establecimientos de educación municipal de la ciudad de Temuco. Se entiende Participación en el Juego como "participar en el juego; mantener un balance entre el juego y las demás ocupaciones; y obtener, utilizar y mantener los juguetes, equipos y suministros apropiadamente". La metodología utilizada para este estudio es de carácter cuantitativo, descriptivo-comparativo, no experimental y de corte transversal. La muestra se selecciona mediante un muestreo no probabilistico intencionado, participando del estudio un total de 68 estudiantes pertenecientes a la Escuela Los Trigales y a la Escuela Campos Deportivos, establecimientos que cuentan con Media Jornada Escolar y Jornada Escolar Completa respectivamente. Los hallazgos de la investigación dan cuenta de un déficit de juego de 8,6 horas semanales, proyectándose un déficit de 344 horas anuales en los niños y niñas que asisten a lornada Escolar Completa, en relación a los escolares que asisten a Media Jornada Escolar. $y$ de diferencias significativas en el balance entre el juego y las demás ocupaciones entre los escolares de ambos establecimientos, dejando en evidencia cómo esta política pública que configura el ambiente institucional educativo en el que se desenvuelven diariam, dejandos escolares, determina sus horatios, ocupaciones disponibles y la distribución de educativo en el que se desenvuelven diariam

\section{Palabras clave}

Políticas Públicas, Educación, Juego e Implementos de Juego.

\section{Abstract}

The research, "Full School Day regimen and Play Participation: A Public Policy in debt with children" shows the impact of Full School Day in Play Participation of schoolchildren of second grade who belong to municipal educational establishments of Temuco city; understanding Play Participation as "participating in the game, maintaining a balance of play with other occupations, and obtain, use and maintain toys, equipment and supplies appropriately". The used metho and non expen Full School Day respectively. The research findings shown a play deficit of 8.6 hours per week, projecting a 344 hours deficit per year for children who attends to Full School Day, in relation to schoolchildren who attend to Half School Day; and significant differences in the balance between play and other occupations, between schoolchildren of both establishments, revealing how this public policy that configures the educational institutional environment in which schoolchildren develop daily, determines their schedules, available occupations and the distribution of activities in their routine.

\section{Keywords}

Public Policies, Education, Play and Playthings.

Recepcionado: 30/03/2017 Aceptado: 01/06/2017

Licenciado en Terapia Ocupacional, Universidad de La Frontera. +56977668049. Email: d.gonzalez22@ufromail.c

Licenciada en Terapia Ocupacional, Universidad de La Frontera. +56956605835. Email: g.leal04@ufromail.cl

Licenciada en Terapia Ocupacional, Universidad de La Frontera. +56987492359. Email: n.monsalves02@ufromail.cl

Licenciada en Terapia Ocupacional, Universidad de La Frontera.+56987492359. Email: k.salazar02@ufromail.cl 


\section{INTRODUCCIÓN}

En 1997 se dictó la Ley № 19.532 que crea el Régimen de Jornada Escolar Completa (JEC) y dicta norma para su aplicación. La puesta en marcha de esta ley significó, entre otras cosas, un aumento significativo de horas cronológicas de clases, sumado a modificaciones curriculares que implican un aumento de exigencia $y$ carga académica; si antes los niños y niñas pasaban alrededor de 5 horas al día en un recinto educacional, hoy, gracias a la implementación de la JEC pasan casi 8 horas, de las cuales tan sólo un aproximado de 1,5 horas corresponden a tiempo libre, tiempo que se distribuye en un período de almuerzo y dos recreos, posicionando a Chile como uno de los países con mayor cantidad de horas de enseñanza anual, encontrándose por sobre la media de los países asociados a la Organización para la Cooperación y el Desarrollo Económico (OCDE) ${ }^{5}$ organización a la cual Chile pertenece.

Según el último informe oficial de Evaluación de la ornada Escolar Completa, emitido por la Dirección de Estudios Sociales de la Pontificia Universidad Católica de Chile (DESUC) ${ }^{6}$, publicado el año 2005, hasta esa fecha existían 6.770 establecimientos educacionales con JEC en el país, que correspondían a una matrícula total de 1.624.848 alumnos. La región de La Araucanía era la segunda región que concentraba el mayor porcentaje de establecimientos en JEC con el $16.9 \%$ del total de establecimientos, antecedida por la décima región con un $20.4 \%$. En dicho informe, además se presentó y analizó la distribución del tiempo escolar a partir de información recogida mediante las fichas de establecimientos educacionales. Los resultados obtenidos arrojaron lo siguiente:

El tiempo total de la Jornada según la norma, corresponde a 35 horas con 25 minutos semanales. Si se observan los tiempos totales que involucra la JEC en los establecimientos, se constata que predominantemente, las escuelas poseían una extensión de la jornada similar a la prevista por la norma $(45,7 \%$ de los casos) o superior a ella (25,3\% de los casos)

Fundada en 1961, la Organización para la Cooperación y el Desarrollo Económico (OCDE) agrupa a 34 países miembros y su misión es promover políticas que mejoren el bienestar económico y socia de las personas alrededor del mundo.

La Dirección de Estudios Sociales de la Pontificia Universidad Católica de Chile (DESUC), desarrolla estudios de opinión pública imagen, servicio, políticas públicas y de tendencias sociales para agencias públicas y medios de comunicación.
En cuanto al tiempo destinado a recreo, el cual según la norma corresponde a 3 horas $10 \mathrm{mi}-$ nutos semanales, se observó una tendencia de los establecimientos a ubicarse bajo el rango esperado para este concepto. Un 45\% de los establecimientos estaban inmediatamente bajo los valores esperados por normativa, y otro $23 \%$ presentaba tiempos de menos de 2 horas y 45 minutos semanales en esta categoría. De los estudiantes, un $46 \%$ consideraba que el tiempo de recreo era menos que suficiente o insuficiente.

- En los cinco años que mediaron entre las dos evaluaciones oficiales a la JEC (2000 - 2005), se produjo un progresivo acortamiento de los tiempos de descanso entre horas de clases. Tanto en enseñanza básica como en la enseñanza media la proporción de establecimientos que estaban en las categorías "crítico" o "preocupante", en relación a los tiempos de descanso entre clases, aumentó significativamente, del 53\% al 68\% en primaria y del $31 \%$ al $63 \%$ en secundaria.

- Cerca de la mitad de las escuelas en JEC (47,8\%), estaban destinando un tiempo pedagógico total muy cercano al esperado según la normativa, correspondiente a 28,5 horas semanales. Otro porcentaje no menor $(26,9 \%)$, estaba destinando tiempos pedagógicos por sobre la norma fijada.

- En dicho informe también se indagó si las escuelas realizaban actividades libres o extracurriculares. Al respecto, se constató que sólo un 17,3\% de las escuelas realizaba estas actividades, las que básicamente remiten a actividades deportivas, recreativas o artísticas. De los estudiantes, un $50 \%$ consideraba como menos que suficiente o insuficientes las horas destinadas a actividades libres o extracurriculares.

Dentro de los datos que aporta la evaluación de la DESUC, queda en evidencia que la implementación de la JEC, a pesar del aumento significativo de horas lectivas, no parece haber disminuido el tiempo de trabajo extraescolar, sino que, al contrario, lo incrementa. En educación básica, un 26\% de los estudiantes destinaba más de dos horas a realizar tareas escolares en sus hogares y un $42 \%$ entre una y dos horas al día (DESUC, 2005). Según el Observatorio Chileno de Políticas Educativas 
$(\mathrm{OPECH}, 2006)^{7}$, comparado con otros países, este alto porcentaje de trabajo escolar en casa suele ser inusual, incluso en países con jornadas escolares más breves. En Europa, se observa que la gran mayoría de los alumnos de 15 años que participaron en el estudio del Programa Internacional de Evaluación de Estudiantes (PISA) 2009 dedicaba menos de dos horas semanales a hacer los deberes o a estudiar en casa. En nueve países, más del 95\% dedicaba menos de dos horas semanales en casa a cada una de las tres materias analizadas (lengua, matemáticas y ciencias) (EACEA; Eurydice; Eurostat, 2012) ${ }^{8}$.

La ley de la JEC se funda sobre la premisa de que más tiempo en clases permite un aprendizaje mayor de los alumnos; sin embargo, la existencia de esta relación no ha podido ser probada de manera consistente en la literatura. Arzola (2011) en el estudio Impacto de la Jornada Escolar Completa en la Evolución del SIMCE, concluye que el tiempo adicional en las aulas, debido a la implementación de la JEC, no se ha traducido en una mejora importante del rendimiento escolar.

A pesar de que no se han evidenciado mejores resultados académicos a raíz de la implementación de la JEC, ésta trajo consigo beneficios sociales que trascienden al ámbito escolar. Ha disminuido el tiempo en que los niños están solos en su casa o en la calle, han aumentado las posibilidades para que la madre trabaje remuneradamente y permite el ahorro del costo de almuerzo de los hijos y de dejar el cuidado de los niños a terceros (Martinic, 2015).

Si bien la Jornada Escolar Completa nace como un programa que pretende mejorar sustantivamente la calidad de la educación en Chile, y que trajo consigo beneficios sociales que trascienden al ámbito escolar, una mejor infraestructura, más recursos y más horas de clases, el balance que se ha hecho hasta ahora no es del todo positivo para los niños y niñas.

En la nota periodística: Niños chilenos juegan seis mil horas menos de lo recomendado, publicada el 18 de octubre de 2015 en el diario La Tercera, edición online,

El Observatorio Chileno de Políticas Educativas (OPECH) es un espacio multidisciplinario de investigación, análisis, difusión y documentación sobre las Políticas Educativas en Chile y nace del trabajo conjunto entre el Equipo de Psicología y Educación de la Universidad de Chile (EPE) y el Programa Interdisciplinario de Investigación en Educación (PIIE).

8 La Red Eurydice tiene como misión analizar y ofrecer información sobre los sistemas y políticas educativas europeas. La componen 37 unidades nacionales con base en los 33 países participantes en el programa de Aprendizaje Permanente de la UE (Estados miembros de la UE, países de la AELC, Croacia y Turquía.
Hartmunt Wedekind ${ }^{9}$ afirma que hasta los siete años un niño debería acumular un total de 15 mil horas de juego, según estándares internacionales. Ilia García ${ }^{10}$ refiere que, en Chile, la cifra apenas Ilega a las nueve mil horas. En el 2011, un estudio Fondecyt advirtió que el "tiempo libre", de los niños de entre 10 y 11 años, eran los trayectos de la casa al colegio y del colegio a la casa. Entre las razones del déficit de juego y de tiempo libre, estaría el aumento de horas de clases, junto con el aumento de la presión académica que trajo consigo la implementación de la Jornada Escolar Completa (González, 2015).

La Convención sobre los Derechos del Niño (UNICEF, $1989)^{11}$ establece que todo niño tiene derecho a educación (Artículo 28), también señala que ésta debe ser orientada para lograr el máximo desarrollo de su personalidad y capacidades (Artículo 29). Sin embargo, también establece que el niño tiene derecho al esparcimiento, al juego y a participar en las actividades artísticas y culturales (Artículo 31), y hoy, gracias a la JEC, los niños no estarían gozando plenamente de este derecho, quedando sin tiempo para distraerse, para jugar... para ser niños.

Para los Terapeutas Ocupacionales este punto no es menor, ya que el juego es considerado el área de la ocupación más relevante en la infancia y debería ser aquella donde los niños inviertan la mayor parte de su tiempo. El juego es conocido por su contribución al desarrollo físico, cognitivo, social y emocional; potencia su autoconcepto, mejora su autoestima y disminuye su frustración en el desempeño de actividades que son más complejas (Blásquez, Guerra, \& Mahmoud-Saleh, 2015). Según Mulligan (2006) es a través del juego que los niños aprenden y practican nuevas habilidades y refinan otras, experimentan con roles sociales, sienten emociones $y$ desarrollan amistades.

Piaget (1966) ha destacado la importancia del juego en los procesos de desarrollo. Ha relacionado el desarrollo de los estadios cognitivos con el desarrollo de la actividad lúdica: las diversas formas de juego que surgen a lo largo del desarrollo infantil son consecuencia directa de las transformaciones que sufren paralelamente las estructuras cognitivas del niño. De los dos componentes

Doctor en Pedagogía y Profesor Diplomado en Matemáticas y Física; Director Científico del Centro de Investigación Infantil Helleum de la Universidad Alice Salomón de Berlín, Alemania.

10 Profesora de Educación Básica; Postgrado en Educación Especial, Diferencial y Administración de Organización Educativa; Gerente general y fundadora Seigard Chile.

11 Fondo de las Naciones Unidas para la Infancia (UNICEF), presente en 191 países, cuya principal finalidad es promover la protección de los derechos de los niños, niñas y adolescentes. 
que presupone toda adaptación inteligente a la realidad (asimilación y acomodación) y el paso de una estructur cognitiva a otra, el juego es paradigma de la asimilación en cuanto que es la acción infantil por antonomasia, la actividad imprescindible mediante la que el niño interacciona con una realidad que le desborda.

Mediante el juego se pueden crear situaciones de máximo valor educativo y cognitivo que permitan ex perimentar, investigar, resolver problemas, descubri y reflexionar. Las implicaciones de tipo emocional, e carácter lúdico, el desbloqueo emocional, la desinhibi ción, son fuentes de motivación que proporcionan una forma distinta a la tradicional de acercarse al aprendizaje (Corbalán \& Deulofeu, 1996).

El juego, al igual que todas las ocupaciones, se desarrolla en un ambiente complejo y multidimensional. Es influenciado por éste y toma significado a través de su contexto físico y sociocultural. El ambiente incluye los espacios que los seres humanos ocupan, los objetos que ellos usan, las personas con quien ellos interactúan y las posibilidades y significados del hacer que existen en el colectivo humano del cual forman parte. Cada ambiente ofrece oportunidades y recursos potenciales, demandas y limitaciones. La oportunidad, el apoyo la demanda y las limitaciones que los aspectos físicos y sociales del ambiente ejercen sobre una persona en particular pueden capacitar o incapacitar al individuo. A menudo, el ambiente es la dimensión crítica que apoya o interfiere con la ocupación de un individuo (Kielhofner 2004). Los ambientes institucionales, sociales, físicos y culturales poseen gran influencia en las personas (Simó \& Urbanowski, 2006)

Bajo esta perspectiva, la Jornada Escolar Completa forma parte del ambiente en el que diariamente los niños y niñas se desempeñan, por lo que podría favorecer o dificultar la Participación en el Juego, entendida como "participar en el juego; mantener un balance entre el juego y las demás ocupaciones; y obtener, utilizar y mantene los juguetes, equipos y suministros apropiadamente" (AOTA, 2014) ${ }^{12}$.

Según la literatura, generalmente los terapeutas se han preocupado primordialmente del medio físico (Simó \& Urbanowski, 2006), omitiendo los aspectos críticos de los ambientes sociales, culturales e institucionales. Asimismo, la intervención se ha centrado en tratamien-

Definición otorgada por la Asociación Americana de Terapia Ocupacional (AOTA) en el Marco de Trabajo para la Práctica de Terapia Ocupacional: Dominio y Proceso, 3ra edición. to y rehabilitación, dejando de lado la promoción y la prevención.

Esta investigación es el primer paso para revelar el impacto de los ambientes institucionales y sociales en la participación en ocupaciones significativas; en este caso la Jornada Escolar de los Establecimientos Educacionales Municipales y su repercusión en la Participación en el Juego de niños y niñas, siendo este el punto de partida para sentar las bases del aporte y la participación activa del profesional de Terapia Ocupacional en la formulación de políticas públicas vinculadas a la educación que resguarden el equilibrio ocupacional entre las diversas áreas en las que los niños y niñas se desempeñan, velando por su desarrollo integral y óptimo.

\section{MÉTODO}

\section{a) Enfoque Metodológico}

El presente estudio es de carácter cuantitativo; descriptivo-comparativo, de la Participación en el Juego de niños y niñas que asisten a Jornada Escolar Completa y la de quienes asisten a Media Jornada Escolar. Es no experimental y de corte transversal, realizándose el segundo semestre del año 2016.

\section{b) Construcción y Justificación de la Muestra}

La población del estudio la componen un total de 99 estudiantes que cursan $2^{\circ}$ año básico, de los cuales 49 pertenecen a la Escuela Los Trigales, establecimiento que cuenta con Media Jornada Escolar en ese nivel, ya que inicia el Régimen de Jornada Escolar Completa en $3^{\circ}$ básico; y 50 pertenecen a la Escuela Campos Deportivos, establecimiento que si cuenta con Jornada Escolar Completa en ese nivel, ya que inicia este régimen en el nivel pre básico.

La selección de la muestra se realiza mediante un muestreo no probabilístico intencionado, en el cual se incluyen todos los niños y niñas que cursen $2^{\circ}$ año básico, cuyas edades se encuentren en un rango de 6 a 9 años, junto a sus padres o apoderados. No se consideran niños y niñas cuyos hogares no se encuentren en el radio urbano de la ciudad de Temuco, por considerar el tiempo de traslado como un factor que también podría tener influencia en la participación en el juego. 
Participan del estudio un total de 68 niños y niñas, de los cuales 14 niños y 19 niñas pertenecen la Escuela Los Trigales, y 18 niños y 17 niñas a la Escuela Campos Deportivos.

Se considera pertinente la muestra para el cumplimiento de los objetivos del estudio, ya que los establecimientos educacionales a los que asisten los escolares se encuentran dentro del radio urbano de la ciudad de Temuco; ambos establecimientos son municipales, por lo que adscriben de manera obligatoria a la Ley de Jornada Escolar Completa, tienen una alta matrícula, son gratuitos y de nivel socioeconómico y rendimiento promedio SIMCE similares.

\section{c) Técnicas de Recolección de Datos}

Se obtiene la información mediante la aplicación de dos instrumentos de recolección confeccionados por el equipo investigador. El primer instrumento consiste en una encuesta cerrada dirigida a los escolares, la cual contiene 6 preguntas, orientadas a las variables de la participación en el juego; el segundo instrumento está dirigido a los padres o apoderados y consiste en una encuesta abierta de 6 preguntas enfocadas a la distribución de los tiempos de Juego, Educación y Descanso en la rutina de los niños y niñas. Ambos instrumentos de recolección de datos se basan en instrumentos propios de la disciplina de Terapia Ocupacional (Historia de Juego de Nancy Takata y Cuestionario Ocupacional, respectivamente).

\section{d) Procedimiento}

Se realiza búsqueda, selección y contacto preliminar con establecimientos municipales de la ciudad de Temuco.

Se accede a la Escuela Los Trigales, establecimiento que inicia la JEC en $3^{\circ}$ año básico, y a la Escuela Campos Deportivos, establecimiento que inicia la JEC en nivel pre escolar. Se coordinan fechas y mecanismos de aplicación de instrumentos. Se aplica consentimiento informado y encuesta a padres y/o apoderados en reunión de curso; posteriormente, en horario de clases, se aplica asentimiento informado y encuesta a los niños y niñas cuyos padres y/o apoderados autorizaron su participación en la investigación.

\section{e) Análisis de Datos}

El procesamiento y análisis de la información estadística se realiza por medio del software SPSS versión 22 . Se utiliza prueba $T$ para muestreo independiente, para el análisis de las respuestas entregadas por los padres y/o apoderados; y prueba Chi-cuadrado para el análisis descriptivo de las respuestas de las preguntas cerradas. El análisis de los datos se lleva a cabo en conjunto por el equipo investigador.

\section{f) Aspectos Éticos}

Se resguardan los derechos y deberes de los participantes, mediante el cumplimiento de la aplicación de Consentimiento y Asentimiento Informado; confidencialidad de la información obtenida y manejo de riesgos en la investigación.

\section{RESULTADOS}

\section{a) Tiempo destinado a Participar en el Juego}

La Figura 1 muestra el tiempo promedio diario destinado por los niños y niñas para jugar. Los escolares que asisten a la Escuela Los Trigales juegan en promedio 3 horas diarias los días de semana y 5,7 horas diarias los fines de semana. Los escolares que asisten a la Escuela Campos Deportivos juegan en promedio 2 horas diarias los días de semana y 3,9 horas diarias los fines de semana.

Existen diferencias estadísticamente significativas en la cantidad de horas que los escolares dedican diariamente a jugar los días de semana, en donde quienes tienen Media Jornada Escolar $(M=3, D E=1.35)$ juegan una hora más que los niños y niñas que asisten a Jornada Escolar Completa $(M=2, D E=1.01)(\mathrm{t}(66)=3.311, \mathrm{p}=0.002)$.

Se encuentran diferencias estadísticamente significativas en la cantidad de horas que los escolares dedican diariamente a jugar los fines de semana, en donde quienes tienen Media Jornada Escolar $(M=5.7, D E=1.9)$ juegan 1,8 horas más que los niños y niñas que asisten a Jornada Escolar Completa $(M=3.9, D E=1.78)(\mathrm{t}(66)$ $=3.593, \mathrm{p}<0.001$ ). 
Figura 1

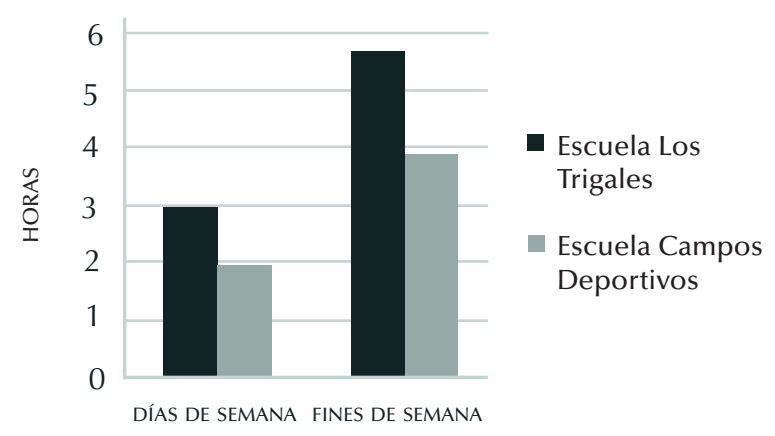

Tiempo promedio diario destinado a jugar

Se obtiene que, del total de los escolares de ambos establecimientos, la mayoría responde que sí les gustaría jugar más, tanto dentro de la escuela $(79,4 \%)$ como fuera de ella $(67,6 \%)$

\section{b) Tiempo destinado a actividades de Educación}

La Figura 2 muestra el tiempo promedio diario destinado por los niños y niñas para estudiar y/o hacer tareas. Los escolares que asisten a la Escuela Los Trigales destinan en promedio 1,3 horas diarias a estudiar o hacer tareas los días de semana y 1,4 horas diarias los fines de semana. Los escolares que asisten a la Escuela Campos Deportivos destinan en promedio 1,3 horas diarias a estudiar o hacer tareas los días de semana y 1,4 horas diarias los fines de semana.

No se encuentran diferencias estadísticamente sig nificativas en la cantidad de horas diarias destinadas estudiar o hacer tareas entre escolares que asisten a Media Jornada Escolar y Jornada Escolar Completa.

No se encuentran diferencias estadísticamente significativas en la cantidad de horas diarias que dedican los escolares a estudiar o hacer tareas en la semana y fines de semana.

\section{c) Tiempo destinado a actividades de Descanso}

La Figura 3 muestra el tiempo promedio diario destinado por los niños y niñas para descansar. Los escolares que asisten a la Escuela Los Trigales descansan en promedio 10 horas diarias los días de semana y 12 horas diarias los fines de semana. Los escolares que asisten la Escuela Campos Deportivos descansan en promedio
Figura 2

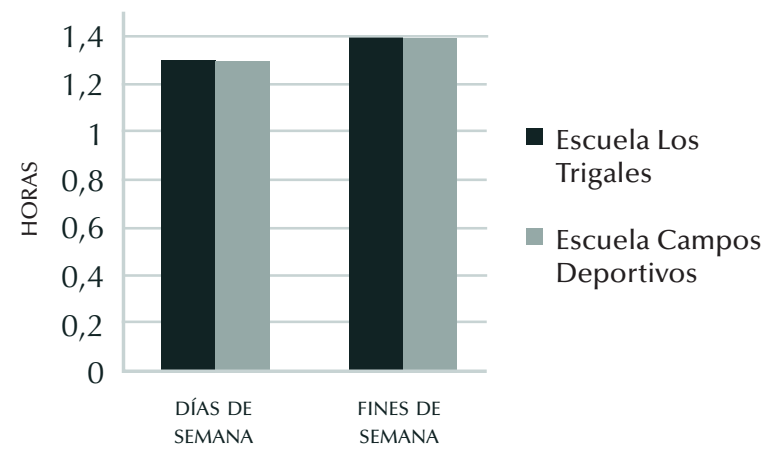

Tiempo promedio diario destinado a estudiar y/o hacer tareas

9 horas diarias los días de semana y 10 horas diarias los fines de semana.

Se encuentran diferencias estadísticamente significativas en la cantidad de horas que los escolares destinan para descansar los días de semana, en donde quienes tienen Media Jornada Escolar $(M=10, D E=1.32)$ descansan una hora más que los niños con Jornada Escolar Completa $M=9, D E=0.95)(\mathrm{t}(66)=3.804, \mathrm{p}<0.001)$.

Se encuentran diferencias estadísticamente significativas en la cantidad de horas que los escolares destinan para descansar los fines de semana, en donde quienes tienen Media Jornada Escolar $(M=12, D E=2.09)$ descansan 2 horas más que los niños con Jornada Escolar Completa $(M=10, D E=1.33)(\mathrm{t}(66)=3.847, \mathrm{p}<0.001)$

\section{d) Tipo de juego predominante}

No existen diferencias estadísticamente significativas en el tipo de juego realizado por los escolares, tanto dentro, como fuera del establecimiento educacional. El juego predominante dentro del establecimiento educaciona de los escolares que asisten a Media Jornada Escolar es de tipo recreativo $(48,5 \%)$, al igual que el de los niños y niñas que asisten a Jornada Escolar Completa $(42,9 \%)$ (chi-cuadrado $=3,02 ; p=0,697$ ); mientras que el juego predominante fuera del contexto escolar de los niños y niñas que asisten a Media Jornada Escolar es de tipo electrónico (39,4\%), al igual que el de los escolares que asisten a Jornada Escolar Completa $(37,1 \%$ ) (chi-cuadrado $=3,28 ; p=0,512$ ). 


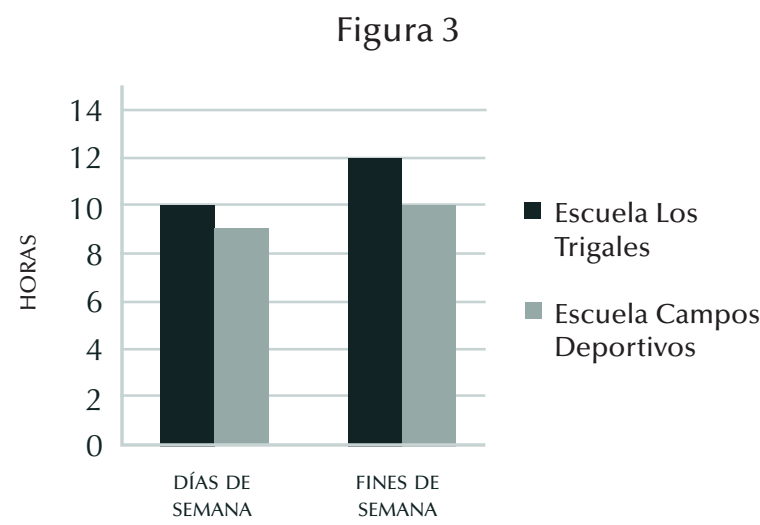

Tiempo promedio diario destinado a descansar

\section{DISCUSIÓN}

Tras el análisis de los resultados, se obtiene que los escolares que asisten a Media Jornada Escolar juegan en promedio 3 horas diarias los días de semana y 5,7 horas diarias los fines de semana, mientras que los niños y niñas que asisten a Jornada Escolar Completa juegan en promedio 2 horas diarias los días de semana y 3,9 horas diarias los fines de semana. Con estos hallazgos se establece un déficit de 8,6 horas semanales y se proyecta un déficit de 344 horas anuales de juego en los niños y niñas que asisten a Jornada Escolar Completa, en relación a los escolares que asisten a Media Jornada Escolar, considerando las 40 semanas lectivas establecidas por ley. Esta información corrobora lo expuesto en la nota periodística Niños chilenos juegan seis mil horas menos de lo recomendado (2015) donde se atribuye el déficit de juego y de tiempo libre, al aumento de horas de clases, junto con el aumento de la presión académica que trajo consigo la implementación de la Jornada Escolar Completa.

Por otra parte, con base en la información aportada por la presente investigación, los escolares que asisten a Media Jornada Escolar destinan en promedio 1,3 horas diarias a estudiar o hacer tareas los días de semana y 1,4 horas diarias los fines de semana, al igual que los que asisten a Jornada Escolar Completa. Esto revela que, independiente del tipo de jornada escolar, los niños y niñas dedican 9,3 horas semanales y se estima un tiempo de 372 horas anuales de trabajo extraescolar, considerando las 40 semanas lectivas. Esto corrobora lo expuesto por el informe de la DESUC (2005) donde se menciona que a pesar del aumento significativo de horas lectivas que trajo consigo la implementación de la Ley JEC, no parece haber disminuido el tiempo de trabajo extraescolar. Dicho informe expone también que, en educación básica, un $26 \%$ de los estudiantes destinaba más de dos horas a realizar tareas escolares en sus hogares y un $42 \%$ entre una y dos horas al día (DESUC, 2005). Tras 12 años de la emisión de este informe, se constata que esta realidad no ha cambiado.

En cuanto a los tiempos de descanso, los niños y niñas que asisten a Media Jornada Escolar descansan en promedio 10 horas diarias los días de semana y 12 horas diarias los fines de semana, mientras que los escolares que asisten a Jornada Escolar Completa descansan en promedio 9 horas diarias los días de semana y 10 horas diarias los fines de semana. Se encuentran diferencias significativas en la cantidad de horas que los escolares destinan para descansar, quedando en evidencia una diferencia de 9 horas semanales y proyectando una diferencia de 360 horas de descanso anuales entre los estudiantes que asisten a Jornada Escolar Completa y Media Jornada Escolar, considerando las 40 semanas lectivas. Estos importantes hallazgos se atribuyen a la impronta de la rutina escolar semanal a la que son sometidos los niños y niñas.

Con la información aportada por la presente investigación, es posible hacer una aproximación de la distribución de los tiempos en la rutina diaria de los niños y niñas de segundo año básico que asisten a Media Jornada Escolar en la Escuela Los Trigales y los escolares que asisten a Jornada Escolar Completa en la Escuela Campos Deportivos, considerando las actividades de Educación, como el tiempo de permanencia en el establecimiento educacional, sumado al tiempo de trabajo extraescolar; las actividades de Descanso, como el tiempo destinado a recostarse sin realizar ninguna actividad en particular, tomar una siesta durante el día y dormir durante la noche; y el tiempo destinado al Juego.

En un día de la semana promedio, un niño o niña que asiste a Media Jornada Escolar destina 6,3 horas de Educación, 10 horas de Descanso y 3 horas de Juego. En tanto, un niño o niña que asiste a Jornada Escolar Completa destina un tiempo de 8,3 horas de Educación, 9 horas de Descanso y 2 horas de Juego; dejando en evidencia que el aumento de horas lectivas y carga académica, obliga a reducir el tiempo destinado a otras ocupaciones.

En un día de fin de semana promedio, un niño o niña que asiste a Media Jornada Escolar destina 1,4 horas de Educación, 12 horas de Descanso y 5,7 horas de Juego. Por otra parte, un niño o niña que asiste a Jornada Escolar Completa destina un tiempo de 1,4 horas de Educación, 10 horas de Descanso y 3,9 horas de Juego; evidenciando 
una significativa diferencia en la distribución de los tiempos destinados a estas ocupaciones entre los escolares según el tipo de Jornada Escolar.

Los resultados de la investigación evidencian un impacto en la Participación en el Juego, principalmente en el tiempo destinado a participar en el juego y en e balance entre el juego y las demás ocupaciones; atribuible al Régimen de Jornada Escolar Completa, que configura el ambiente institucional educativo en el que se desenvuelven diariamente los escolares, determinando sus horarios, ocupaciones disponibles y la distribución de las actividades en su rutina.

Teniendo como antecedente lo expuesto en la presente investigación, es imperioso determinar las consecuencias que este impacto implicaría en los niños y niñas, en sus dimensiones física, cognitiva, social y emocional, tanto a corto como a largo plazo, y por qué no, indagar en a incidencia que esto podría tener en el aumento de diagnósticos de patologías de salud mental en los niños y niñas los últimos años, como por ejemplo, el trastorno por déficit atencional con hiperactividad (TDAH).

La intención de los investigadores no es hacer una crítica al Régimen de Jornada Escolar Completa para retornar al sistema de Media Jornada Escolar, ya que la implementación de la JEC conlleva beneficios que no son netamente educativos, sino que se enmarcan en el ámbito social. El equipo investigador plantea la necesidad de incorporar profesionales terapeutas ocupacionales que participen activamente en la elaboración de Proyectos Educativos Institucionales en los establecimientos; en la creación, revisión y reformulación de Políticas Públicas de educación, incorporando una visión más amplia de proceso educativo, considerando el juego como eje fundamental del desarrollo integral de los niños y niñas; en la implementación de metodologías de enseñanza más lúdicas, redistribución de los tiempos pedagógicos y de descanso, y diseño de espacios físicos adecuados en los establecimientos para el desarrollo óptimo del juego. Junto con eso, concientizar a padres, madres, docentes, paradocentes, directivos y sociedad en general sobre la relevancia del juego en el desarrollo de los niños y niñas, de tal manera que se incorpore de manera armónica entre las ocupaciones en las que participan diariamente.

Por último, el equipo investigador extiende la invitación a ampliar los horizontes del quehacer del terapeuta ocupacional, de modo que el actuar del profesional trascienda más allá del tratamiento y rehabilitación en un déficit ya instaurado, y asuma un rol protagónico en la promoción de la salud y en la prevención de limitaciones en la participación y desempeño ocupacional asociadas a factores ambientales, y no sólo físicos, sino que también sociales, culturales e institucionales.

\section{RefERENCIAS BIBLIOGRÁfiCAS}

American Occupational Therapy Association. (2014). Occupational therapy practice framework: Domain and process. American Journal of Occupational Therapy, 68(1), S1-S48. Recuperado de http://dx.doi org/10.5014/ajot.2014.682006

Arzola, M. P. (2011). Impacto de la Jornada Escolar Completa en la Evolución del SIMCE. Libertad y Desarrollo. Recuperado de http://repositorio. minedu.gob.pe/handle/123456789/4325

Blásquez, M. P., Guerra, L., \& Mahmoud-Saleh, L. (2015). Terapia Ocupacional Pediátrica: Algo más que un juego. Revista Terapia Ocupacional Galicia, 12(7), 100-114. Recuperado de http://www.revistatog.com/ mono/num7/pediatrica.pdf

Corbalán, F., Deulofeu, J. (1996). Juegos manipulativos en la enseñanza de las matemáticas. UNO, revista de Didáctica de las Matemáti$\operatorname{cas}(7), 71-80$.

DESUC. (2005). Evaluación de la Jornada Escolar Completa. Pontificia Universidad Católica de Chile, Dirección de Estudios Sociales. Recuperado de http://www.opech.cl/bibliografico/Participacion_Cultura_Escolar/Informe_final_jec.pdf

EACEA; Eurydice; Eurostat. (2012). Cifras claves de la educación en Europa 2012. Bruselas: Eurydice. doi:10.2797/86046

González, C. (18 de Octubre de 2015). Niños chilenos juegan seis mil horas menos de lo recomendado. La Tercera. Recuperado de http:// www.latercera.com/noticia/ninos-chilenos-juegan-seis-mil-horas-menos-de-lo-recomendado/

Kielhofner, G. (2004). Modelo de Ocupación Humana. Buenos Aires, Argentina: Editorial Médica Panamericana.

Martinic, S. (2015). El Tiempo y el Aprendizaje Escolar: La experiencia de la Jornada Escolar en Chile. Revista Brasileira de Educação 20(61), 479-499. Recuperado de https://dx.doi.org/10.1590/S141324782015206110

Ministerio de Educación. (13 de noviembre de 1997). Crea el Régimen de Jornada Escolar Completa diurna y dicta normas para su aplicación [Ley № 19.532].

Mulligan, S. (2006). Terapia Ocupacional en Pediatría: Proceso de evaluación. Madrid, España: Editorial Médica Panamericana.

OPECH. (2006). Jornada Escolar Completa: La Divina Tragedia de La Educación Chilena. Observatorio Chileno de Políticas Educativas. Recuperado de http://www.opech.cl/inv/documentos_trabajo/JEC.pd Piaget, J. (1990). La formación del símbolo en el niño. México: Fondo de Cultura Económica.

Simó, S., \& Urbanowski, R. (2006). El Modelo Canadiense de Desempeño Ocupacional. Revista Terapia Ocupacional Galicia(3), 1-27. Recuperado de http://www.revistatog.com/num3/pdfs/Expertol.pdf

UNICEF (1989). Convención sobre los Derechos del Niño Recuperado de http://www.unicef.cl/web/informes/derechos_nino/convencion.pdf 